\title{
Noncompaction in Healthy Subjects, Dilated and Hypertrophic Cardiomyopathy, and Neuromuscular Disorders Is the Same Entity
}

\author{
Claudia Stollberger ${ }^{1, *} ;$ Josef Finsterer $^{1}$ \\ ${ }^{1}$ Medical Department With Cardiology and Intensive Care Medicine Krankenanstalt Rudolfstiftung, Vienna, Austria \\ ${ }^{*}$ Corresponding author: Claudia Stollberger, Medical Department With Cardiology and Intensive Care Medicine Krankenanstalt Rudolfstiftung, Vienna, Austria. Tel/Fax: $+43-$ \\ 19454291, E-mail: claudia.stoellberger@chello.at
}

Received: May 14, 2014; Accepted: January 16, 2015

Keywords: Cardiomyopathies; Child; Echocardiography; Left Ventricles

With interest we read the article by Khosroshahi et al. about a novel method for quantification of left ventricular hypertrabeculation/noncompaction (LVHT) using two-dimensional echocardiography in children (1). We appreciate their efforts to contribute to an improvement and unification of echocardiographic diagnostic criteria for LVHT, which is urgently needed. Concerning their proposed method, we have the following questions and concerns:

We are confused about the echocardiographic views in which the measurements should be performed. In the methods it is described that the percentages of LVHT were measured at three short-axis levels. However, in the mentioned figure, only the apical level is a short-axis view. At the papillary level, the outline of the left ventricle is oval and thus not a short-axis view. At the mitral valve level, the section is a modified apical 4 -chamber view. Additionally we want to ask how it was possible to exactly delineate the left ventricular cavity from the surface of the trabeculations, a border which is extremely irregular and variable and thus difficult to determine even on a two-dimensional plane? The examples given in the mentioned figures showed that the tracing lines were coarse and did not follow each of the trabeculations. Additionally, it remains uncertain how the area was measured in the original article number $1 \mathrm{~A}$ and $1 \mathrm{~B}$ since the tracing lines cannot be seen in the region of the posterior wall. Thus we want to know if the authors assessed inter- and intraobserver variability of the measurements.

How were the images for measurement selected? Were they selected by the same investigator who performed the measurements? Which was the transducer frequency used for the investigations? LVHT is usually not present at the mitral valve level. Why was this level chosen for measurement? We want to know if there was any correlation between left ventricular systolic function or left ventricu- lar end diastolic diameter and percentages of LVHT.

The authors recommend a threshold value of $17 \%$ to distinguish between LVHT and dilated cardiomyopathy. Is this value the same in all three levels of measurement or does it relate to the average? It is mentioned that 2 children with dilated cardiomyopathies had a LVHT $>17 \%$. Did these 2 patients also fulfill other diagnostic criteria for LVHT?

Was it possible to compare the echocardiographic findings in any of the included patients with pathoanatomic or intraoperative investigations of the heart? Was cardiac magnetic resonance imaging carried out in any of the included patients and was there any correspondence between echocardiographic and magnetic resonance findings?

Regarding the diagnosis of LVHT, it may not only be overlooked but also overdiagnosed. Papillary muscles, aberrant bands and chordae tendineae might pretend LVHT, especially in the short-axis views. Thus, we want to know how the authors differentiated these structures from LVHT. In some cases LVHT is not congenital and develops after birth (2). Was LVHT present already on previous echocardiographies?

In how many LVHT-patients, dilative cardiomyopathy patients and controls did echocardiography show endocardial fibrosis? Endocardial fibrosis is a frequent finding in LVHT and might contribute to its recognition (3).

Which segments of the left ventricle were most frequently affected in LVHT-patients, dilative cardiomyopathy patients and controls? What was the ethnicity of the included patients and controls? There are indications in the literature that the amount of trabeculations is variable among ethnicities (4).

How many of the included patients suffered from neuromuscular disorders, which are reported to be frequently associated with LVHT and influence the prog-

Copyright (C) 2015, Growth \& Development Research Center. This is an open-access article distributed under the terms of the Creative Commons Attribution-NonCommercial 4.0 International License (http://creativecommons.org/licenses/by-nc/4.0/) which permits copy and redistribute the material just in noncommercial usages, provided the original work is properly cited. 
nosis of adults with LVHT (5)? Were follow-up investigations carried out and did LVHT percentage change over time?

Though interesting the approach by Khosroshahi et al. (1) has some major limitations. The reference limit of $17 \%$ cannot be drawn from only four cases, it was not tested if the reference limit also applies for adults, and it was not tested if the reference limit depends on sex or ethnicity, no interobserver variability was calculated, and no comparison of the sensitivity/specificity with other diagnostic methods was carried out. These diagnostic criteria need to be evaluated on a larger cohort of LVHT patients and all limitations need to be addressed.

\section{Authors' Contributions}

Claudia Stollberger: concept, design, acquisition of data, data analysis/interpretation, drafting of the manuscript, critical revision of the manuscript and approval of the article; Josef Finsterer: concept, acquisition of data, data analysis/interpretation and critical revision of the manuscript and approval of the article.

\section{References}

1. A novel method for quantification of left ventricular noncompaction using two-dimensional echocardiography in children. Khosroshahi AG, Zanjani KS, Kocharian A, Zeinaloo A, Kiani A, Rad EM. Iran J Pediatr. 2013;23(5):519.

2. Acquired left ventricular noncompaction as a cardiac manifestation of neuromuscular disorders. Finsterer J, Stollberger C, Schubert B. Scand Cardiovasc J. 2008;42(1):25

3. Pathomorphologic findings in left ventricular hypertrabeculation/noncompaction of adults in relation to neuromuscular disorders. Gerger D, Stollberger C, Grassberger M, Gerecke B, Andresen H, Engberding R, et al. Int J Cardiol. 2013;169(4):249.

4. Quantification of left ventricular trabeculae using fractal analysis. Captur G, Muthurangu V, Cook C, Flett AS, Wilson R, Barison A, et al. J Cardiovasc Magn Reson. 2013;15:36.

5. Neurological comorbidity affects prognosis in left ventricular hypertrabeculation/noncompaction. Stollberger C, Blazek G, Wegner C, Finsterer J. Heart Lung. 2012;41(6):594. 